\title{
Revealing Hund's multiplets in Mott insulators under strong electric fields
}

\author{
Nagamalleswararao Dasari $\odot,{ }^{1,}{ }^{*}$ Jiajun Li $\odot,{ }^{1}$ Philipp Werner, ${ }^{2}$ and Martin Eckstein ${ }^{1, \dagger}$ \\ ${ }^{1}$ Department of Physics, University of Erlangen-Nuremberg, 91058 Erlangen, Germany \\ ${ }^{2}$ Department of Physics, University of Fribourg, 1700 Fribourg, Switzerland
}

(Received 4 July 2019; revised manuscript received 20 March 2020; accepted 20 March 2020; published 10 April 2020)

\begin{abstract}
We investigate the strong-field dynamics of a paramagnetic two-band Mott insulator using real-time dynamical mean-field theory. We demonstrate that strong electric fields can lead to a transient localization of electrons. This nonequilibrium quantum effect allows us to reveal specific signatures of local correlations in the time-resolved photoemission spectrum. In particular, we demonstrate that the localization can be strong enough to produce atomiclike spin multiplets determined by the Hund's coupling $J$, and thus provide a way of measuring $J$ inside the solid. Our simulation also fully incorporates nonlinear field-induced tunneling processes, which would lead to a dielectric breakdown in the steady state limit. A careful analysis of these processes, however, shows that they remain weak enough and do not prevent the measurement of the transiently localized spectra.
\end{abstract}

DOI: 10.1103/PhysRevB.101.161107

Introduction. The interplay between orbital degeneracy and strong interactions is the origin of some of the most spectacular phenomena in correlated materials. Embedding atomiclike multiplets which are determined by the Hund's coupling $J$ and other local interactions into the solid gives rise to a plethora of interesting phenomena, including superconductivity or magnetism driven by spin or orbital fluctuations, strange metallic behavior, and metal-insulator transitions [1]. Hund's multiplets can also leave interesting signatures in the ultrafast dynamics [2,3].

Accurate estimates of local interactions are hard to obtain from first principles [4,5], and multiplets are often not directly visible through spectroscopy on the solid. Following the rapid progress in the control of solids with ultrashort laser pulses $[6,7]$, it is therefore an obvious question whether nonequilibrium probes can provide different means to uncover the local interactions. An intriguing gedanken experiment is to perform a spectroscopic measurement while electrons are transiently localized by switching off the tunneling to neighboring atoms. This could be realized by photoemission in the presence of a strong time-periodic field, which is known to renormalize the tunneling through dynamical localization [8]. However, the driving frequency would have to be both large compared to the relevant energy scales (interaction, bandwidth) and avoid resonant interband transitions. This constraint is already difficult to realize in cold atom experiments [9], and even more so in solids. We thus pose the question whether experiments in the opposite regime, i.e., using a subcycle resolved measurement of the spectrum for driving frequencies far below the Mott gap, can realize a similar transient localization.

The basic mechanism we hope to exploit in this way is field-induced localization (FIL), i.e., the transient localization of electrons in a potential energy gradient. FIL is well

\footnotetext{
*nagamalleswararao.d@gmail.com

${ }^{\dagger}$ martin.eckstein@fau.de
}

understood for noninteracting systems, where single-particle wave functions become exponentially localized in a potential gradient, with equally spaced energy levels (Wannier-Stark ladder). The effect has been observed in bulk GaAs [10], and the Wannier-Stark localization was proposed to enhance polaronic effects in organic crystals [11], or induce a dimensional crossover [12]. For correlated electrons, however, there is no simple analytical understanding of the bandwidth renormalization (in contrast to dynamical localization). Moreover, strong fields in Mott insulators have an antagonistic effect, field-induced tunneling (FIT), where charge excitations in the insulator are created due to the field [13-17] (the analog of the Zener breakdown [18] in band insulators). Such excitations may mask the spectra of transiently localized electrons, similar to electron-electron scattering washing out the WannierStark ladder in metals $[19,20]$. In this Rapid Communication we study a setting which captures both FIL and FIT, and demonstrate that correlation effects in transiently localized spectra can be observed for realistic values of the Mott gap and bandwidth. The necessary atomically strong electric fields on the femtosecond timescale have been already used to explore intriguing phenomena, including Bloch oscillations, LandauZener tunneling [21,22], and the electric field control of spin [23].

Model and method. We study an orbitally degenerate Mott insulator using the two-orbital Hubbard model

$$
H=-\sum_{i, j, l l^{\prime} \sigma} e^{i \phi_{i j}(t)} c_{i l \sigma}^{\dagger} \hat{T}_{l l^{\prime}}^{i j} c_{j l^{\prime} \sigma}+\sum_{i} H_{i}^{\mathrm{loc}}
$$

where $c_{i l \sigma}^{\dagger}$ creates an electron at the lattice site $i$ in an orbital $l \in\{1,2\}$ with spin $\sigma \in\{\uparrow, \downarrow\}$. The $\hat{T}^{i j}$ are the hopping matrices along the bonds $(i j)$, and the electric field enters in Eq. (1) as a time-dependent Peierls phase $\phi_{i j}=e\left(\vec{R}_{i}-\vec{R}_{j}\right) \vec{A}(t) / \hbar c$, where $\vec{A}(t)=-c \int_{0}^{t} d s \vec{E}(s)$ is the vector potential. The local 
term contains the Kanamori interaction [24]

$$
\begin{aligned}
H_{i}^{\mathrm{loc}}= & U \sum_{l} n_{i l \uparrow} n_{i l \downarrow}+\sum_{\sigma, \sigma^{\prime}, l \neq l^{\prime}}\left(U^{\prime}-J \delta_{\sigma \sigma^{\prime}}\right) n_{i l \sigma} n_{i l^{\prime} \sigma^{\prime}} \\
& +J \sum_{l \neq l^{\prime}}\left(c_{i l \uparrow}^{\dagger} c_{i l \downarrow}^{\dagger} c_{i l^{\prime} \downarrow} c_{i l^{\prime} \uparrow}+c_{i l \uparrow}^{\dagger} c_{i l^{\prime} \downarrow}^{\dagger} c_{i l \downarrow} c_{i l^{\prime} \uparrow}\right),
\end{aligned}
$$

where $U$ and $U^{\prime}=(U-2 J)$ are the intra- and interorbital Coulomb interactions, respectively, and $J$ is the Hund's coupling. For two electrons, the local multiplet energies $U+J$, $U-J$, and $U-3 J$ are split by $2 J$.

We use real-time dynamical mean-field theory (DMFT) $[25,26]$ to solve this model. While the results of this Rapid Communication should hold qualitatively for a generic lattice structure, we employ the specific setting introduced in Ref. [27], which allows for a closed form of the DMFT selfconsistency relation: This involves a Bethe lattice in which each site reflects the local environment of an atom in a cubic lattice with $e_{g}$ orbitals $l=d_{x^{2}-y^{2}}, d_{3 z^{2}-r^{2}}$, and in which the electric field acts along the body diagonal [28]. The DMFT equations for the local orbital-dependent Green's function $G_{l, l^{\prime}}\left(t, t^{\prime}\right)$, and the current $j(t)$ are identical to Ref. [27], and therefore repeated only in the Supplemental Material [29]. The largest hopping matrix element $t_{0}=1$ sets the energy scale, so that the free half bandwidth is $W=2 t_{0}$. Furthermore, we set $\hbar=1$ (time is measured in units of $\hbar / t_{0}$ ), and $e=1$, i.e., the field is measured in units of $t_{0} / e a$, where $a$ is a lattice constant. Unless otherwise stated, for all results the system is initially in equilibrium at temperature $T=0.1$ (paramagnetic phase), the Hubbard interaction is $U=8$ (Mott insulator), and the total filling of the system $n_{\text {tot }}=1$ (quarter filling).

Field-induced tunneling. We first characterize the FIT which may potentially compete with the observation of localized spectra. We smoothly ramp on a field of amplitude $E_{0}$ within a time $t_{r}=15$. The results do not depend on the chosen ramp profile as long as $E(t)$ has negligible frequency content at the Mott gap $U$ (to avoid direct absorption), which is the case for $t_{r} \ll 1 / U$ [30]. The time-dependent electric current $j(t)$ and the fraction of doubly occupied sites $\delta d(t) \equiv$ $[d(t)-d(0)]$ is plotted in Fig. 1. Similar to the analogous setting in the one-orbital case [15] we can distinguish two regimes, (i) and (ii), as described below:

(i) When $E_{0}$ is sufficiently smaller than the gap, the current saturates to a nonzero constant value after a peak during the switch-on of the field $\left(E_{0}=1.6\right.$ in Fig. 1). The initial peak corresponds to the build-up of a polarization in the insulator, while the almost constant current at later times amounts to processes ("FIT") in which an electron tunnels over $\ell$ sites and thereby gains the energy $\Delta_{g}$ to create a doublon-holon pair $\left(\ell \propto \Delta_{g} / E_{0}\right)$. Correspondingly, after the switch-on, $d(t)$ increases with a rate proportional to $j / E_{0}$. The tunneling mechanism suggests that the current is proportional to $j / E_{0} \propto$ $\exp \left(-E_{\mathrm{th}} / E_{0}\right)$, where the threshold field $E_{\mathrm{th}}$ should scale with the gap. This has been confirmed for the single-band Mott insulator [13,15]. Here, we demonstrate this behavior in Fig. 2(a) by plotting $j / E_{0}$ (measured at late times when the current is steady) logarithmically against $1 / E_{0}$. Linear fits (dashed lines) indicate the exponential behavior, and $E_{\mathrm{th}}$ is extracted from the slope [see Fig. 2(c)]. For given $J$, the threshold field increases with $U$, due to an increase in the

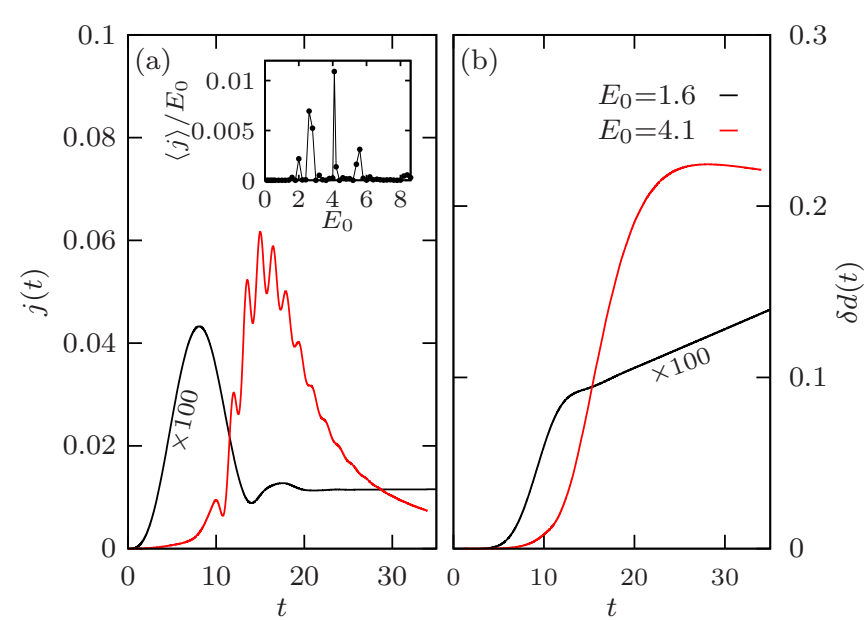

FIG. 1. (a) Electric current and (b) double occupancy for different field strengths $E_{0}$ at $J=0$. Inset: Current averaged over times $25 \leqslant t \leqslant 35$ as a function of $E_{0}$.

Mott gap. With increasing $J, E_{\mathrm{th}}$ decreases linearly $E_{\mathrm{th}} \approx$ $a(U-b J)$ as expected from the dependence of the Mott gap on $J$ [1]. A rough understanding of this behavior is obtained from the multiplets in the atomic limit. Due to the exponential dependence on the gap, the tunneling rate is dominated by the smallest multiplet excitation $U-3 J$. For a nonzero bandwidth, the parameter $b \approx 5.4,5.2,5$ for $U=6,7,8$ turns out to be different from 3 , but has the correct sign and becomes closer to 3 with increasing $U$. For further confirmation, we have also performed an analogous analysis in the half-filled

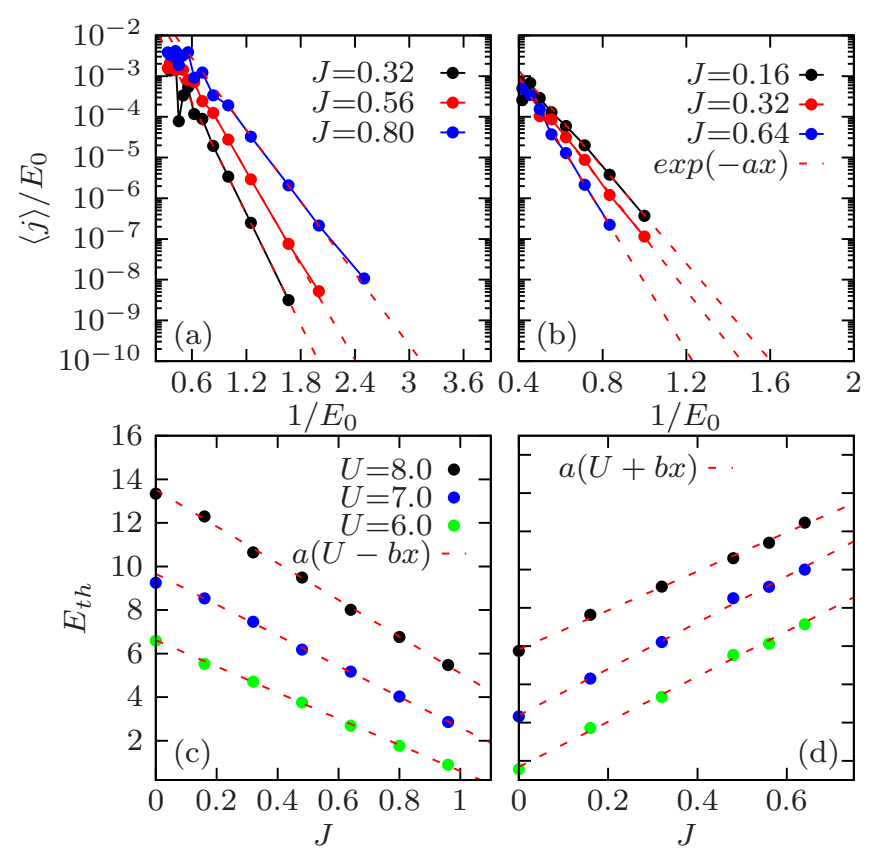

FIG. 2. Upper panel: Current (averaged over the times $25<t<$ 35 ) in the Mott insulator for two different fillings: (a) $n_{\text {tot }}=1$ and (b) $n_{\text {tot }}=2$. Lower panels: The threshold field $E_{\text {th }}$ extracted from the exponential fit for $E_{0} \lesssim 1$ (dashed lines in the upper panel), plotted as a function of $J$ for (c) $n_{\text {tot }}=1$ and (d) $n_{\text {tot }}=2$. 
Hubbard model $\left(n_{\text {tot }}=2\right)$ [see Figs. 2(b) and 2(d)]. In this case, the system is initially predominantly in the high-spin doublon state (energy $U-3 J$ ), and tunneling creates a singly occupied and a triply occupied state with excitation energy $(3 U-5 J)-2(U-3 J)=U+J$, which is consistent with an increase of $E_{\text {th }}$ with $J$ [Fig. 2(d)].

(ii) For larger fields, a strong enhancement of the fieldinduced excitation is observed. The doublon density quickly saturates to a large value, after which the current decays to zero $\left(E_{0}=4.1\right.$ in Fig. 1$)$. Similar to the one-band Hubbard model, at $J=0$ this rapid excitation occurs at resonances $U=n E_{0}$ with a small integer $n$ (inset in Fig. 1). In the twoband model, once sites become doubly occupied by the field, resonances $2 U=n E_{0}$ become visible due to the generation of triply occupied sites from doubly occupied sites. Due to the broadening of the resonances and the quick heating of the system to infinite temperature, the $J$ dependence of these resonances is hard to resolve and unlikely to provide a good experimental path to extract local physics. We have therefore shifted a detailed analysis to the Supplemental Material [29], and in the following focus on the observation of local correlation effects in the transient spectral functions.

Spectral functions. For the same protocol as above, we have calculated the time-dependent single-particle spectral functions $A(t, \omega)=-\operatorname{Im} \int_{0}^{s_{\max }} d s G^{R}(t, t-s) e^{i \omega s}$ for different field strengths. Here, $G^{R}=\sum_{l} G_{l l}^{R}$ is the orbitally averaged local propagator, and $s_{\max }$ a cutoff set by the simulation time. In equilibrium, the spectrum has a clear Mott gap [Fig. 3(a)]. For very large values of $J$, the upper Hubbard band, which corresponds to transitions from predominantly singly occupied sites to the doublon multiplets, is split into three peaks separated by $2 J$, but this multiplet structure is no longer visible when $J$ is sufficiently smaller than the bandwidth. When the field is turned on [Fig. 3(b) for $J=0$ ], we observe the emergence of Wannier-Stark states at energy shifts $n E_{0}$ from the main Hubbard bands at $\omega=\mu, U+\mu$ (and also from the weak higher-order Hubbard bands at $\omega \approx$ $2 U-\mu, 3 U-\mu)$, together with a band narrowing of the central peak which manifests FIL. (The band at $\omega=2 U-\mu$ mainly corresponds to the insertion of an electron to a doubly occupied site, which has a substantial amplitude only once such doublons have been induced by field-induced tunneling, while the resonance at $\omega=3 U-\mu$ corresponds to the simultaneous creation of a triply occupied site and a holon upon insertion of an electron, which is possible even in equilibrium due to virtual charge fluctuations.) Weak Hund's coupling $J$ [Fig. 3(c)] broadens the sidebands, and eventually splits all peaks emerging from the Hubbard band into multiplets separated by $2 J$. This is shown clearly by three line plots of the upper Hubbard band for different electric fields [see Figs. 3(d)-3(f)]. The splitting is observed for intermediate $E_{0}=3.2$, but disappears for large fields $\left(E_{0} \approx U\right)$ where the strong excitation broadens the bands. Due to the band narrowing the multiplet splitting of the Hubbard bands is observed at much smaller values of $J$ than in the equilibrium case.

The above analysis indicates that FIL can be used to measure the multiplets spectroscopically, if a strong field can be maintained for long enough without substantial FIT. In a realistic setup for measuring the doublon multiplets,
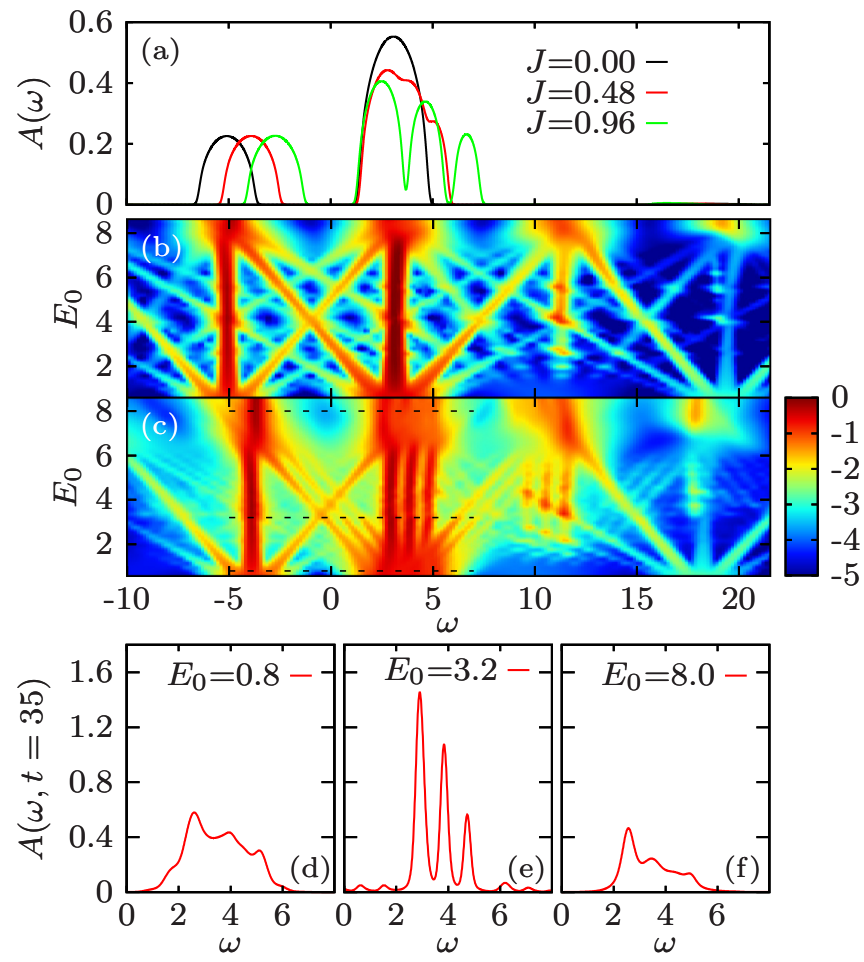

FIG. 3. (a) Equilibrium single-particle spectral function at $U=$ 8 for different values of $J$. (b) and (c) Intensity map of the timedependent single-particle spectral functions $\left[\log _{10}|A(\omega)|\right]$ plotted for (b) $J=0$ and (c) $J=0.48$. Lower panel (d)-(f): Line plots of singleparticle spectral functions plotted for the field strengths shown by the dashed lines in (c).

the Mott insulator could be exposed to strong $\mathrm{THz}$ pulses with frequencies much smaller than the Mott gap. Here, we simulate a drive of the system with a single-cycle pump pulse of the form

$$
E_{\mathrm{pu}}(t)=E_{0} \sin \left[\omega_{p}\left(t-t_{0}\right)\right] e^{-4.6\left(t-t_{0}\right)^{2} / t_{0}^{2}},
$$

and then calculate the time-resolved photoemission spectrum (trPES) at different delay times $t_{d}$, for a Gaussian probe pulse with envelope $S(t)=\exp \left(\frac{-t^{2}}{2 t_{c}^{2}}\right)$ that is sufficiently shorter than the time period of the pump, using the standard expression [31] $I\left(\omega, t_{d}\right) \propto-i \iint d t d t^{\prime} S\left(t-t_{d}\right) S\left(t^{\prime}-\right.$ $\left.t_{d}\right) e^{i \omega\left(t-t^{\prime}\right)} G^{<}\left(t, t^{\prime}\right)$. In the quarter-filled case $\left(n_{\text {tot }}=1\right)$, FIL becomes manifest through a narrowing of the lower band and a splitting of the upper band, following the time profile of the field $\left|E_{\mathrm{pu}}(t)\right|$ of the pump pulse [Fig. 4(a)]. The upper Hubbard band corresponds to unoccupied states in equilibrium and is therefore only revealed after it has acquired some occupation due to FIT during the pulse itself. In a three-quarter-filled system $\left[n_{\text {tot }}=3\right.$, Fig. 4(b)], multiplets are also seen below the Fermi energy, i.e., in the lower Hubbard band, which corresponds to transitions from mainly triply occupied sites into the doublon manifold [Fig. 4(b)]. Note that the multiplet position does not shift during the pulse, which shows that there is no measurable field-induced renormalization of the multiplet energies.

Optical response. The multiplet splitting observed in the spectrum suggests that similar signatures resulting from transitions between the various sidebands may be observ- 


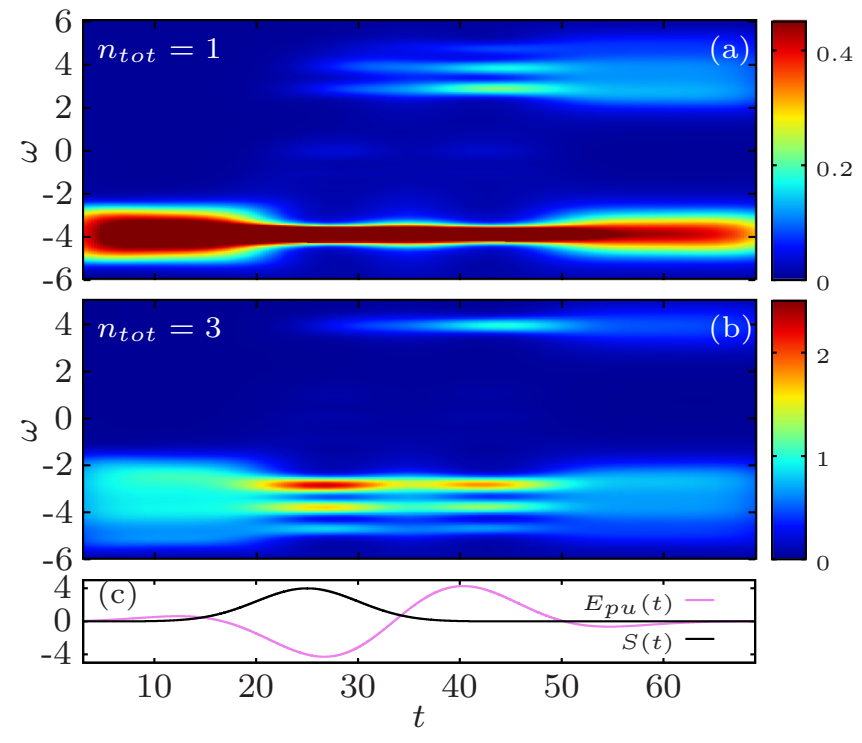

FIG. 4. Time-dependent photoemission spectrum for (a) quarter filling and (b) three-quarter filling. The pump pulse is plotted in (c), together with a probe envelope $S(t)$ of duration $t_{c}=5$ in arbitrary units. The interaction parameters are $U=8, J=0.48$, the amplitude of the pump pulse is $E_{0}=5.4$, and $\omega_{p}=0.18$.

able in the (experimentally more easily accessible) optical response. We have calculated the optical conductivity of the Mott insulator in a pump-probe setup using the expression [32] $\sigma(\omega)=\frac{J_{\mathrm{pr}}(\omega)}{E_{\mathrm{pr}}(\omega)}$, where $J_{\mathrm{pr}}(\omega)=J_{\mathrm{pr}+\mathrm{pu}}(\omega)-J_{\mathrm{pu}}(\omega)$ is the difference in the current measured with and without the probe field $E_{\mathrm{pr}}(\omega)$. The pump profile is given by the electric field used in the FIT, and the probe is $E_{\mathrm{pr}}(t)=A_{0} e^{-\left(t-t_{d}\right)^{2} / 2 t_{c}^{2}}(t-$ $\left.t_{d}\right) / t_{c}^{2}$, with pulse duration $t_{c}$ and delay $t_{d} ; A_{0}$ is weak enough to ensure we probe the linear response. Figure 5 shows $\sigma(\omega)$ for three different values of the pump field $E_{0}$.

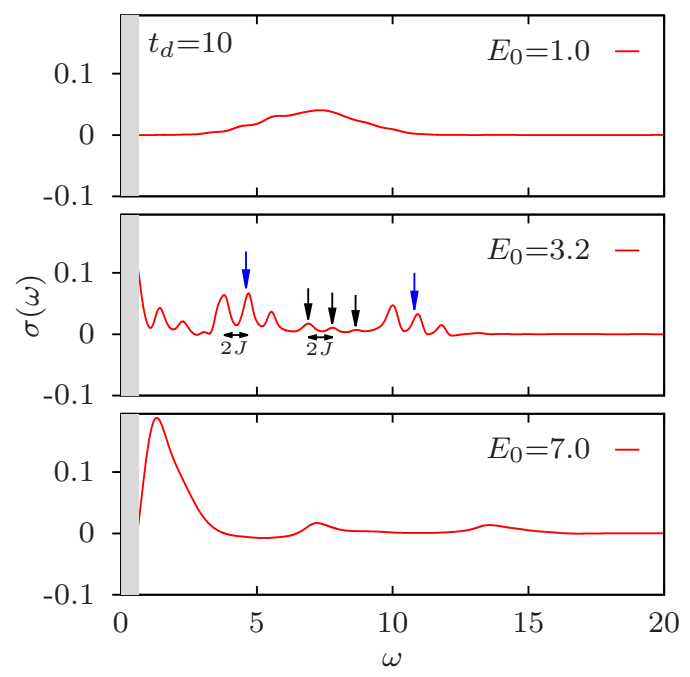

FIG. 5. Optical conductivity of the Mott insulator plotted for different field strengths. The arrows indicate the multiplet excitations. The parameters are $U=8$ and $J=0.48$. Same field profile $E(t)$ as in Fig. 3, probe at delay $t_{d}=10$.
For small $E_{0}$, there is a broad Hubbard band around $\omega \approx 7$ [Fig. 5(a)]. A three-peak structure (shown by black arrows) with a separation of $2 J$ between the peaks becomes visible for moderate field strengths [Fig. 5(b)]. The peaks highlighted by blue arrows represent the transitions from the lower Hubbard band to Wannier-Stark sidebands of the doublon multiplets. Because in the optical excitation, intersite transitions parallel (opposite) to the field direction are dominant, the $2 J$-split Wannier-Stark sidebands of the Hubbard band are much stronger than the main Hubbard band in the optical conductivity. The peaks for $\omega \lesssim 4$ correspond to inter-Hubbard-band transitions, which become possible once the system is excited. The optical conductivity around the Hubbard bands finally becomes flat for large fields $\left[E_{0} \approx U\right.$, Fig. 5(c)] due to the increase of the effective temperature of the system. This shows that $\sigma(\omega)$ is more strongly affected by excitations through FIT than the trPES, but multiplet signatures in the optical conductivity can be expected in a regime of moderately strong fields. We have also performed simulations with transient $\mathrm{THz}$ pulses, as in Fig. 4, and confirmed that multiplet signatures become visible for suitable parameters.

Conclusion. We have studied a two-band Mott insulator in strong electric field transients. The results demonstrate that strong fields can transiently localize electrons in Mott insulators, so that spectroscopic probes such as trPES can reveal local interaction effects. To utilize this field-induced localization (FIL) in real materials, it is important that the antagonistic field-induced tunneling (FIT) remains weak, because a large density of excitations can destroy the Mott state, renormalize interactions through dynamic screening [33], or generate Mott-Hubbard excitons [34,35], which would complicate the analysis. Both FIT and FIL nontrivially depend on the Mott gap and the bandwidth, and the present nonequilibrium DMFT approach can nonperturbatively treat both effects. We observe an exponentially activated tunneling current (analogous to the one-orbital case), but in a large parameter regime the tunneling rate is sufficiently weak that the spectra of transiently localized electrons can be probed long enough to reach high energy resolution. This provides a general path to reveal local correlation effects in the solid, and to measure interaction parameters which are often hard to obtain from first-principles simulations. The present model focuses on the Hund's coupling $J$, which is the dominant local interaction in many transition metal oxides, but one can anticipate that spin-orbit coupling, crystal field effects, and local phonon couplings can also be resolved when they are of sufficient magnitude. While the present model simulation intends to establish the effect in principle, and material-specific simulations would be needed to establish the optimal pulse duration and amplitude to balance FIT and FIL for a given material, the parameters of our model are in the realistic range for transition-metal monoxides such as $\mathrm{MnO}, \mathrm{FeO}, \mathrm{CoO}$, and $\mathrm{NiO}$, with a Coulomb interaction comparable to the bandwidth, and $J$ a factor $3-5$ smaller [36,37]. Experimentally, it is challenging to perform time-resolved angle-resolved photoemission spectroscopy (trARPES) experiments in the presence of strong fields (due to the ponderomotive effect of the field on the photoemitted electrons), but the recent realization of such an experiment [38] shows that the observation of transient electron localization is within in reach. 
Acknowledgments. This work was supported by the ERC Starting Grant No. 716648. The calculations have been done at the RRZE of the University Erlangen-Nuremberg. P.W. acknowledges support by ERC Consolidator Grant No. 724103.

[1] A. Georges, L. de' Medici, and J. Mravlje, Annu. Rev. Condens. Matter Phys. 4, 137 (2013).

[2] H. U. R. Strand, D. Golež, M. Eckstein, and P. Werner, Phys. Rev. B 96, 165104 (2017).

[3] J. Rincón, E. Dagotto, and A. E. Feiguin, Phys. Rev. B 97, 235104 (2018).

[4] C. Honerkamp, H. Shinaoka, F. F. Assaad, and P. Werner, Phys. Rev. B 98, 235151 (2018).

[5] F. Aryasetiawan, M. Imada, A. Georges, G. Kotliar, S. Biermann, and A. I. Lichtenstein, Phys. Rev. B 70, 195104 (2004).

[6] D. N. Basov, R. D. Averitt, and D. Hsieh, Nat. Mater. 16, 1077 (2017).

[7] C. Giannetti, M. Capone, D. Fausti, M. Fabrizio, F. Parmigiani, and D. Mihailovic, Adv. Phys. 65, 58 (2016).

[8] D. H. Dunlap and V. M. Kenkre, Phys. Rev. B 34, 3625 (1986).

[9] M. Weinberg, C. Ölschläger, C. Sträter, S. Prelle, A. Eckardt, K. Sengstock, and J. Simonet, Phys. Rev. A 92, 043621 (2015).

[10] C. Schmidt, J. Bühler, A.-C. Heinrich, J. Allerbeck, R. Podzimski, D. Berghoff, T. Meier, W. G. Schmidt, C. Reichl, W. Wegscheider, D. Brida, and A. Leitenstorfer, Nat. Commun. 9, 2890 (2018).

[11] P. Werner and M. Eckstein, Europhys. Lett. 109, 37002 (2015).

[12] C. Aron, G. Kotliar, and C. Weber, Phys. Rev. Lett. 108, 086401 (2012).

[13] T. Oka and H. Aoki, Phys. Rev. Lett. 95, 137601 (2005).

[14] T. Oka and H. Aoki, Phys. Rev. B 81, 033103 (2010).

[15] M. Eckstein, T. Oka, and P. Werner, Phys. Rev. Lett. 105, 146404 (2010).

[16] S. Kirino and K. Ueda, J. Phys. Soc. Jpn. 79, 093710 (2010).

[17] B. Mayer, C. Schmidt, A. Grupp, J. Bühler, J. Oelmann, R. E. Marvel, R. F. Haglund, T. Oka, D. Brida, A. Leitenstorfer, and A. Pashkin, Phys. Rev. B 91, 235113 (2015).

[18] T. Oka, R. Arita, and H. Aoki, Phys. Rev. Lett. 91, 066406 (2003).

[19] S. Mandt, Phys. Rev. A 90, 053624 (2014).

[20] M. Eckstein and P. Werner, Phys. Rev. B 84, 035122 (2011).

[21] O. Schubert, M. Hohenleutner, F. Langer, B. Urbanek, C. Lange, U. Huttner, D. Golde, T. Meier, M. Kira, S. W. Koch, and R. Huber, Nat. Photonics 8, 119 (2014).
[22] T. Higuchi, C. Heide, K. Ullmann, H. B. Weber, and P. Hommelhoff, Nature (London) 550, 224 (2017).

[23] T. Kampfrath, A. Sell, G. Klatt, A. Pashkin, S. Mährlein, T. Dekorsy, M. Wolf, M. Fiebig, A. Leitenstorfer, and R. Huber, Nat. Photonics 5, 31 (2010).

[24] J. Kanamori, Prog. Theor. Phys. 30, 275 (1963).

[25] J. K. Freericks, V. M. Turkowski, and V. Zlatić, Phys. Rev. Lett. 97, 266408 (2006).

[26] H. Aoki, N. Tsuji, M. Eckstein, M. Kollar, T. Oka, and P. Werner, Rev. Mod. Phys. 86, 779 (2014).

[27] J. Li, H. U. R. Strand, P. Werner, and M. Eckstein, Nat. Commun. 9, 4581 (2018).

[28] Fields directed along a certain crystalline direction can imply a partial field localization, or dimensional crossover [12].

[29] See Supplemental Material at http://link.aps.org/supplemental/ 10.1103/PhysRevB.101.161107 for more details about hopping matrices and the expression for current, and for more details about current resonances and the excitation density of doublons, which includes Ref. [39].

[30] The precise field profile for $t<t_{r}$ is $E(t)=$ $E_{0}\left[\frac{1}{2}-\frac{3}{4} \cos \left(\frac{\pi t}{t_{r}}\right)+\frac{1}{4} \cos \left(\frac{\pi t}{t_{r}}\right)^{3}\right]$.

[31] J. K. Freericks, H. R. Krishnamurthy, and T. Pruschke, Phys. Rev. Lett. 102, 136401 (2009).

[32] C. Shao, T. Tohyama, H.-G. Luo, and H. Lu, Phys. Rev. B 93, 195144 (2016).

[33] D. Golež, M. Eckstein, and P. Werner, Phys. Rev. B 92, 195123 (2015).

[34] K. Zawadzki and A. E. Feiguin, Phys. Rev. B 100, 195124 (2019).

[35] N. Bittner, D. Golež, M. Eckstein, and P. Werner, Phys. Rev. B 101, 085127 (2020).

[36] L. Zhang, P. Staar, A. Kozhevnikov, Y.-P. Wang, J. Trinastic, T. Schulthess, and H.-P. Cheng, Phys. Rev. B 100, 035104 (2019).

[37] X. Ren, I. Leonov, G. Keller, M. Kollar, I. Nekrasov, and D. Vollhardt, Phys. Rev. B 74, 195114 (2006).

[38] J. Reimann, S. Schlauderer, C. P. Schmid, F. Langer, S. Baierl, K. A. Kokh, O. E. Tereshchenko, A. Kimura, C. Lange, J. Güdde, U. Höfer, and R. Huber, Nature (London) 562, 396 (2018).

[39] M. Eckstein and P. Werner, Phys. Rev. B 82, 115115 (2010). 